\title{
Hepatitis $C$ testing and re-testing among people attending sexual health services in Australia, and hepatitis $C$ incidence among people with human immunodeficiency virus: analysis of national sentinel surveillance data
}

David C. Boettiger ${ }^{1 *}$, Matthew G. Law ${ }^{1}$, Gregory J. Dore ${ }^{1}$, Rebecca Guy ${ }^{1}$, Denton Callander ${ }^{1}$, Basil Donovan ${ }^{1,2}$, Catherine C. O'Connor ${ }^{3,4}$, Christopher K. Fairley ${ }^{5}$, Margaret Hellard ${ }^{6}$ and Gail Matthews ${ }^{1}$

\begin{abstract}
Background: Direct acting antivirals are expected to drastically reduce the burden of hepatitis $\mathrm{C}$ virus (HCV) in people living with Human Immunodeficiency Virus (HIV). However, rates of HCV testing, re-testing and incident infection in this group remain uncertain in Australia. We assessed trends in HCV testing, re-testing and incident infection among HIV-positive individuals, and evaluated factors associated with HCV re-testing and incident infection.

Methods: The study population consisted of HIV-positive individuals who visited a sexual health service involved in the Australian Collaboration for Coordinated Enhanced Sentinel Surveillance (ACCESS) between 2007 and 2015. Poisson regression was used to assess trends and to evaluate factors associated with HCV re-testing and incident HCV infection.
\end{abstract}

Results: There were $9227 \mathrm{HIV}$-positive individuals included in our testing rate analysis.

Of 3799 HIV-positive/HCV-negative people that attended an ACCESS sexual health service more than once, 2079 (54.7\%) were re-tested for $\mathrm{HCV}$ and were therefore eligible for our incidence analysis. The rate of HCV testing increased from 17.1 to 51.4 tests per 100 patient years between 2007 and 2015 ( $p$ for trend <0.01). Over the same period, HCV re-testing rates increased from 23.9 to 79.7 tests per 100 person years ( $p$ for trend $<0.01$ ). A clear increase in testing and re-testing began after 2011. Patients who identified as men who have sex with men and those with a history of injecting drug use experienced high rates of HCV re-testing over the course of the study period. Among those who re-tested, 157 incident HCV infections occurred at a rate of 2.5 events per 100 person years. Between 2007 and 2009, 2010-2011, 2012-2013 and 2014-2015, rates of incident HCV were $0.8,1.5,3.9$ and 2.7 events per 100 person years, respectively ( $p$ for trend $<0.01$ ). Incident HCV was strongly associated with a history of injecting drug use.

Conclusions: High rates of HCV testing and re-testing among HIV-positive individuals in Australia will assist strategies to achieve HCV elimination through rapid treatment scale up. Continued monitoring of HCV incidence in this population is essential for guiding both HCV prevention and treatment strategies.

Keywords: Hepatitis C virus, Human immunodeficiency virus, Sexual health, Australia

\footnotetext{
* Correspondence: dboettiger@kirby.unsw.edu.au

'The Kirby Institute, UNSW Sydney, Sydney, NSW, Australia

Full list of author information is available at the end of the article
} 


\section{Background}

Hepatitis $\mathrm{C}$ virus (HCV) is a major contributor to morbidity and mortality in people living with Human Immunodeficiency Virus (HIV) [1-3]. In Australia, it is estimated that around 3000 individuals are $\mathrm{HIV} / \mathrm{HCV}$ co-infected [4, 5]. Interferon-based therapies for $\mathrm{HCV}$ have exhibited poor efficacy and significant toxicity in this population, limiting the capacity for large scale treatment delivery and success. However, current evidence indicates that direct acting antivirals (DAAs) exhibit excellent safety and efficacy in HIV-positive and HIV-negative individuals [6]. Moreover, the first DAAs became available under government subsidy to all $\mathrm{HCV}$ infected Australian adults in March 2016 [7].

Australia has one of the highest rates of HCV screening globally with an estimated $80 \%$ of the HCV infected population having been tested and diagnosed [5]. While current guidelines recommend HCV antibody testing for all HIV-positive people at initial presentation and at least annually thereafter (with a follow up HCV RNA test to confirm chronic infection if antibody testing is positive) [8], true rates of testing and incident infection in this group remain uncertain.

To allow monitoring of changes in $\mathrm{HCV}$ incidence after the introduction of DAA therapies it is important to evaluate past incidence trends. This will also facilitate the development of mathematical models to investigate the likely impact of DAA treatment scale-up and identify factors that could reduce the anticipated benefit of DAAs. Such work could have substantial public health implications as it is anticipated that appropriate use of DAAs in the HIV-positive population will lead to eventual elimination of $\mathrm{HIV} / \mathrm{HCV}$ co-infection [9].

This paper assesses trends in HCV testing and re-testing among people attending Australian sexual health services, and evaluates trends in incident $\mathrm{HCV}$ infection among HIV-positive individuals. It also explores factors associated with $\mathrm{HCV}$ re-testing and incident infection among HIV-positive individuals.

\section{Methods}

\section{Study population}

The study population consisted of patients attending sexual health services participating in the Australian Collaboration for Coordinated Enhanced Sentinel Surveillance (ACCESS) project. ACCESS is a HIV/sexually transmitted infection sentinel surveillance network of sexual health services, general practice clinics, infectious diseases hospital outpatient clinics, and pathology laboratories. The methods behind this collaboration have been described in detail elsewhere [10]. For this analysis, de-identified demographic, behavioral, and clinical data were extracted from the patient management systems of 42 sexual health services using customized extraction scripts. Services were located in New South Wales, Victoria, Western Australia, Queensland, and the Northern Territory.

The ACCESS sexual health services network is broadly representative of sexual health services across the country. In Australia, sexual health services frequently manage the care of men who have sex with men (MSM) and people living with HIV. Nevertheless, a recent study of gay and bisexual men attending ACCESS general practice clinics found that about half were tested for a sexually transmitted infection between 2011 and 2015 [5]. The current analysis precedes a wider investigation of $\mathrm{HCV}$ testing and incidence across both sexual health services and general practice clinics which is currently being planned.

\section{Data analysis}

Data on patient demographics, HIV and HCV testing were analyzed for patients who attended an ACCESS sexual health service between 2007 and 2015. Three main analyses were undertaken: (i) trends in HCV testing; (ii) trends in, and factors associated with, HCV re-testing; and (iii) trends in, and factors associated with, incident $\mathrm{HCV}$ infection. $\mathrm{HCV}$ testing/re-testing could consist of $\mathrm{HCV}$ antibody or RNA testing, and HCV positivity could include a positive $\mathrm{HCV}$ antibody or RNA test. Incident $\mathrm{HCV}$ infection was defined as a negative antibody test followed by either a positive antibody test or a positive RNA test, or a negative RNA test followed by a positive RNA test. Factors associated with $\mathrm{HCV}$ testing were not evaluated because of the bias introduced by high-risk individuals knowing their HCV status before attending an ACCESS sexual health service and therefore not undergoing testing.

HCV testing: Patients were included in the HCV testing rate population if they had at least one clinic visit after their first visit and were not known to be HCV-positive. Baseline was defined as the date of the first clinic visit. Annual rates of HCV testing were stratified by HIV status (HIV-negative individuals were retained as a comparison group). Follow up was censored at the time of testing HCV-positive, at HIV seroconversion, or 3 months after the last clinic visit. Those that were censored because of HIV seroconversion could re-enter the analysis as long as they had documentation of at least one further clinic visit after their date of HIV seroconversion.

$\mathrm{HCV}$ re-testing: Patients were only included in the $\mathrm{HCV}$ re-testing rate population if they had documentation of a negative HCV test and at least one further clinic visit. Baseline was defined as the date the first negative $\mathrm{HCV}$ test was undertaken. Annual rates of re-testing were stratified by HIV status. Censoring was as per the testing rate analysis. Those that were censored because of HIV seroconversion could re-enter the analysis as long as they had 
documentation of a negative HCV test while HIV-positive and at least one further clinic visit.

$\mathrm{HCV}$ incidence: HIV-positive individuals in our retesting rate analysis were included in our HCV incidence population if they had at least one $\mathrm{HCV}$ test after a negative $\mathrm{HCV}$ antibody test, or at least one HCV RNA test after a negative HCV RNA test. The date of $\mathrm{HCV}$ infection was taken as the mid-point between the last negative $\mathrm{HCV}$ test and the first positive $\mathrm{HCV}$ test. Follow up was censored 3 months after the last negative HCV test for those that never returned a positive result.

Statistics: Poisson regression was used to assess the significance of time trends in $\mathrm{HCV}$ testing, re-testing and incidence, and to evaluate factors associated with $\mathrm{HCV}$ re-testing and incident $\mathrm{HCV}$ infection. Our analysis of factors associated with $\mathrm{HCV}$ re-testing was split by calendar year (between 2007 and 2011 and between 2012 and 2015). Baseline age, sex, country of birth, place of residence, sexual orientation, history of sex work (at any stage of study period), history of injecting drug use (at any stage of study period), and Aboriginal and Torres Strait Islander status were considered fixed covariates. CD4 cell count, HIV viral load, antiretroviral therapy use and calendar year were evaluated as time-updated covariates. Undetectable HIV viral load was defined as $<200$ copies $/ \mathrm{ml}$. Covariates were considered for inclusion in our multivariate models if they exhibited a univariate $p$-value $<0.2$. They were retained in the multivariate models if they exhibited an adjusted $p$-value $<0.05$. Patients with missing data for any of the covariates analyzed were included in all analyses, but incidence rate ratios (IRRs) for missing covariate categories are not reported. Stata (StataCorp, College Station, TX) version 14.1 was used for all statistical analysis.
Sensitivity analyses: We chose to include HCV RNA in our primary definitions of $\mathrm{HCV}$ testing/re-testing and incident $\mathrm{HCV}$ infection to account for the fact that $\mathrm{HCV}$ testing is often not performed as recommended [11]. Others have defined incident infection as seroconversion only $[12,13]$. To enhance the comparability of our findings we repeated our main analyses using only antibody testing/re-testing and seroconversion as our definitions of $\mathrm{HCV}$ testing/re-testing and $\mathrm{HCV}$ positivity, respectively.

\section{Results}

Of 362,341 patients who attended a sexual health service contributing data to ACCESS, there were 198,050 who contributed follow up time in our testing rate analysis. However, since 2073 patients contributed data to both our HIV-positive and HIV-negative study populations (i.e., they seroconverted during follow up), the total number of HIV-negative and HIV-positive individuals contributing to each group was 190,896 and 9227, respectively. In our re-testing rate analysis, 25,248 patients contributed follow up - 21,945 HIV-negative and 3799 HIV-positive. Of the 3799 HIV-positive patients in our re-testing rate analysis, 2079 were re-tested at least once for HCV after their initial negative test and were therefore included in our incidence analysis. Figure 1 provides a comprehensive breakdown of the patient selection process.

\section{HCV testing}

Baseline characteristics of the $\mathrm{HCV}$ testing rate population (HIV-positive and HIV-negative) are presented in Additional file 1: Table S1. In the HIV-positive group, median age was 40 years (interquartile range [IQR] 31-48)

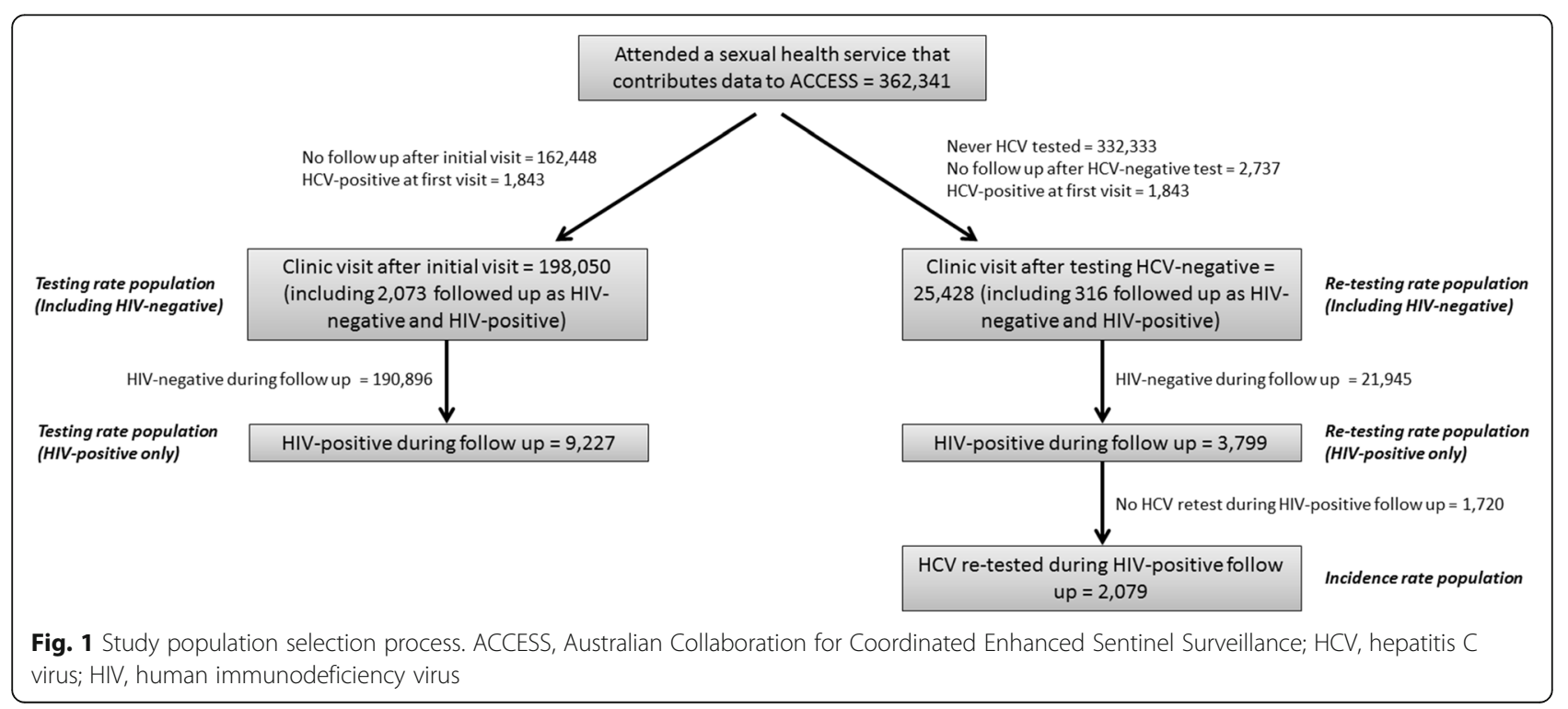


and $85.5 \%$ were male. In the HIV-negative group, median age was 27 years [IQR 23-36] and $58.0 \%$ were male.

Figure 2 shows that, among HIV-positive individuals, the rate of $\mathrm{HCV}$ testing increased from 17.1 tests per 100 person years (95\% confidence interval [CI] $15.2-$ 19.1 ) in 2007 to 51.4 tests per 100 person years (95\% CI $49.2-53.8$ ) in 2015 ( $p$ for trend <0.01). In HIV-negative individuals, the rate increased from 6.5 tests per 100 person years (95\% CI 6.1-6.9) in 2007 to 15.2 tests per 100 person years $(95 \% \mathrm{CI} 14.6-15.8)$ in 2015 ( $p$ for trend $<0.01$ ). A steady increase in $\mathrm{HCV}$ testing began after 2011 in both groups.

Similar trends were found in our sensitivity analyses focusing on $\mathrm{HCV}$ antibody testing. In HIV-positive individuals, the rate of $\mathrm{HCV}$ antibody testing increased from 7.9 tests per 100 person years (95\% CI 6.7-9.3) to 39.4 tests per 100 person years $(95 \%$ CI 37.4-41.4) between 2007 and 2015 ( $p$ for trend <0.01). Among HIV-negative individuals over the same period, the rate increased from 2.3 tests per 100 person years $(95 \%$ CI $2.1-2.6)$ to 11.1 tests per 100 person years $(95 \%$ CI $10.6-11.6$; $p$ for trend $<0.01)$.

\section{HCV re-testing}

Baseline characteristics of the $\mathrm{HCV}$ re-testing rate population (HIV-positive and HIV-negative) are presented in Additional file 1: Table S2. Among the HIV-positive group, median age was 39 years (IQR 30-48) and 88.2\% were male. In the HIV-negative group, median age was 29 years [IQR $23-38$ ] and $61.7 \%$ were male.

In HIV-positive individuals, the rate of $\mathrm{HCV}$ re-testing increased from 23.9 tests per 100 person years $(95 \%$ CI 17.9-32.0) in 2007 to 79.7 tests per 100 person years
(95\% CI 75.7-83.8) in 2015 ( $p$ for trend <0.01). Among HIV-negative individuals, the rate increased from 26.5 tests per 100 person years (95\% CI 23.5-29.8) in 2007 to 52.4 tests per 100 person years (95\% CI 49.6-55.3) in 2015 ( $p$ for trend $<0.01$ ). An increase in HCV re-testing began after 2011 (see Fig. 3).

Table 1 shows that, among HIV-positive individuals, a history of injecting drug use and identifying as MSM were the only factors significantly associated with a shorter time to HCV re-testing between both 2007 2011 and 2012-2015. Country of birth other than Australia, residing in a regional/remote location, and identifying as Aboriginal or Torres Strait Islander were associated with a greater rate of $\mathrm{HCV}$ re-testing between 2007 and 2011 and younger age, residing in a major city, and later calendar year were associated with a greater rate of HCV re-testing between 2012 and 2015.

Similar results were found in our sensitivity analyses. In HIV-positive individuals, the rate of $\mathrm{HCV}$ re-testing increased from 21.2 tests per 100 person years (95\% CI $13.3-33.6)$ to 76.9 tests per 100 person years $(95 \% \mathrm{CI}$ 72.2-81.8) between 2007 and 2015 ( $p$ for trend $<0.01$ ). Among HIV-negative individuals over the same period, the rate increased from 17.9 tests per 100 person years (95\% CI 13.9-23.2) to 49.3 tests per 100 person years (95\% CI 45.9-52.9; $p$ for trend <0.01). A history of injecting drug use and identifying as MSM remained important factors associated with HCV re-testing between both 2007-2011 (IRR 2.1, 95\% CI 1.6-2.9, $p<0.01$ for history of injecting versus no history and IRR 1.8, 95\% CI 1.2-2.9, $p=0.01$ for MSM versus heterosexual male) and 2012-2015 (IRR 1.2, 95\% CI 1.0-1.5, $p=0.09$ for

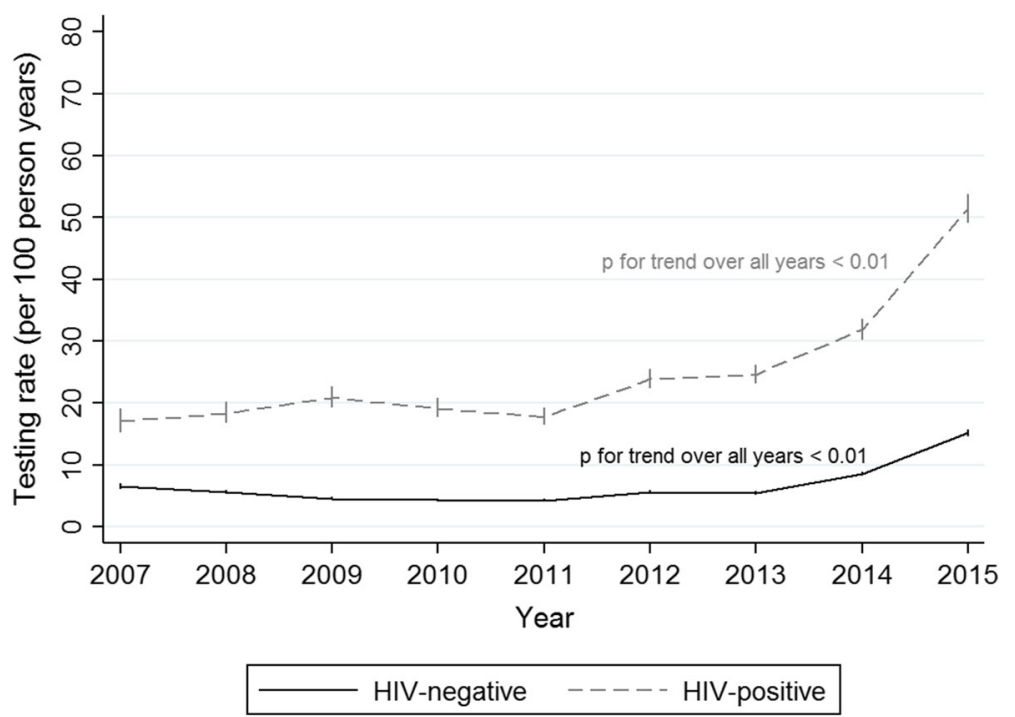

Fig. 2 Rates of HCV testing in HIV-positive $(n=9227)$ and HIV-negative $(n=190,896)$ people. There were 2073 individuals who HIV seroconverted during follow up and contributed person years to both HIV-positive and HIV-negative study populations. Error bars represent $95 \%$ confidence interval. HCV, hepatitis C virus; HIV, human immunodeficiency virus 


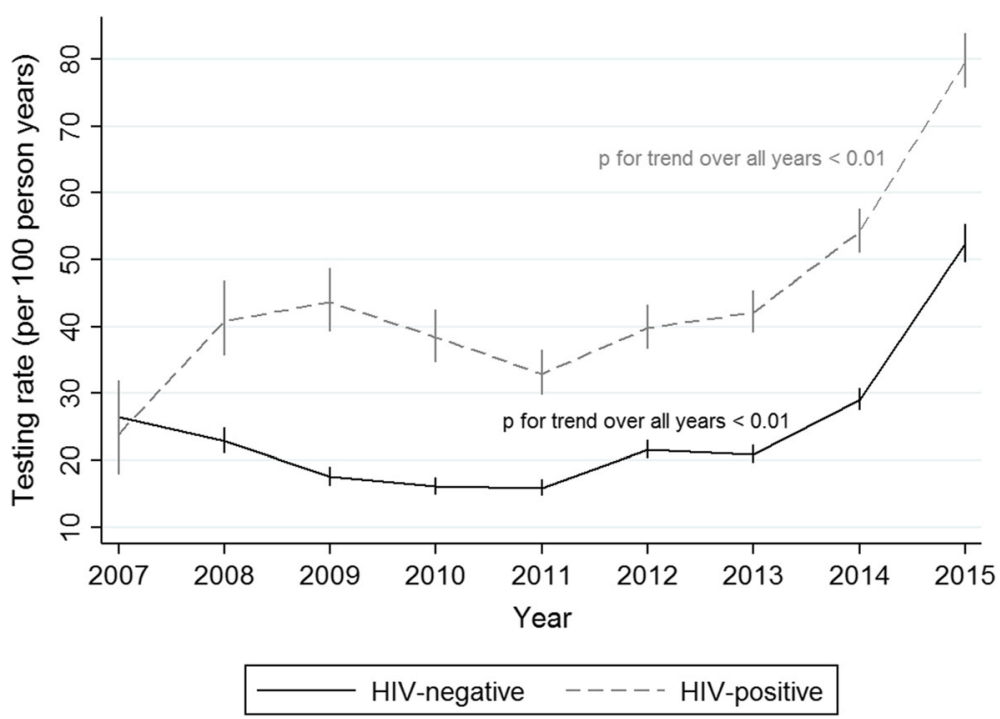

Fig. 3 Rates of HCV re-testing in HIV-positive $(n=3799)$ and HIV-negative $(n=21,945)$ people. There were 316 individuals who HIV seroconverted during follow up and contributed person years to both HIV-positive and HIV-negative study populations. Error bars represent $95 \%$ confidence interval. HCV, hepatitis C virus; HIV, human immunodeficiency virus

history of injecting versus no history and IRR 1.3, 95\% CI 1.1-1.6, $p=0.01$ for MSM versus heterosexual male). Those residing in a major city were also subject to a higher rate of re-testing between 2012 and 2015 (IRR $0.7,95 \%$ CI $0.6-0.8, p<0.01$ for regional/remote versus major city).

\section{HCV incidence}

Characteristics of the $\mathrm{HCV}$ incidence rate population at the time of their first negative HCV test are presented in Additional file 1: Table S3. The median age was 41 years (IQR 32-50) and $90.7 \%$ were male. Median CD4 cell count was 510 cells $/ \mathrm{mm}^{3}$ (IQR $356-672$ ), $74.9 \%$ of patients with a known treatment status were using antiretroviral therapy, and $50.2 \%$ of patients with a documented HIV viral load had a level that was undetectable.

Over the study period, 157 incident $\mathrm{HCV}$ infections occurred at a rate of 2.5 events per 100 person years (95\% CI 2.1-2.9). Between 2007 and 2009, 2010-2011, 2012-2013 and 2014-2015, rates of incident HCV were 0.8 (95\% CI 0.4-1.6), 1.5 (95\% CI 1.0-2.3), 3.9 (95\% CI 3.1-4.9) and 2.7 (95\% CI 2.0-3.5) events per 100 person years, respectively ( $p$ for trend $<0.01$ ). During the same periods, rates among those who identified as MSM without a history of injecting drug use were 0.4 (95\% CI 0.1-1.4), 1.2 (95\% CI 0.6-2.1), 3.0 (95\% CI 2.2-4.1) and 2.5 (95\% CI 1.8-3.5) events per 100 person years, respectively ( $p$ for trend $<0.01$ ). Among MSM with a history of injecting drug use, rates were 1.8 (95\% CI 0.57.4), 2.6 (95\% CI 1.0-6.7), 10.7 (95\% CI 6.9-16.3) and 2.7 (95\% CI 1.1-6.6) events per 100 person years, respectively $(p$ for trend $=0.24)$. In our final model adjusted for calendar year, incident HCV infection was associated with younger age, living in a major city, being a heterosexual male, having a history of injecting drug use, and being an Aboriginal or Torres Strait Islander (Table 2). When sexual orientation and injecting drug use were included as an interaction term, there was no difference in the rate of incident $\mathrm{HCV}$ infection between non-injecting MSM and non-injecting male heterosexuals (IRR 1.4 with non-injecting MSM as the reference group, 95\% CI $0.8-2.4, p=0.25$ ), but a substantial difference between injecting MSM and injecting male heterosexuals (IRR 3.1 with injecting MSM as the reference group, 95\% CI 1.5-6.1, $p<0.01$ ).

In our sensitivity analysis focusing on $\mathrm{HCV}$ antibody testing, 14 incident $\mathrm{HCV}$ infections occurred at a rate of 0.4 events per 100 person years (95\% CI 0.2-0.6). Between 2007 and 2009, 2010-2011, 2012-2013 and 2014-2015, rates of incident $\mathrm{HCV}$ were 0.2 (95\% CI 0.0-1.6), 0.1 (95\% CI 0.0-1.0), 0.6 (95\%CI 0.3-1.2) and 0.3 (95\% CI $0.1-0.8)$ events per 100 person years, respectively ( $p$ for trend $=0.59$ ). The low number of seroconversions observed precluded us from developing a meaningful multivariate model, however, a history of injecting drug use was strongly associated with incident HCV infection in our univariate model (IRR 8.4, 95\% CI $2.9-24.2, p<0.01$ versus no history).

\section{Discussion}

$\mathrm{HCV}$ testing and re-testing in Australian sexual health services has increased substantially among HIV-positive (and HIV-negative) people since 2011. Rates of retesting were consistently high among MSM and injecting 


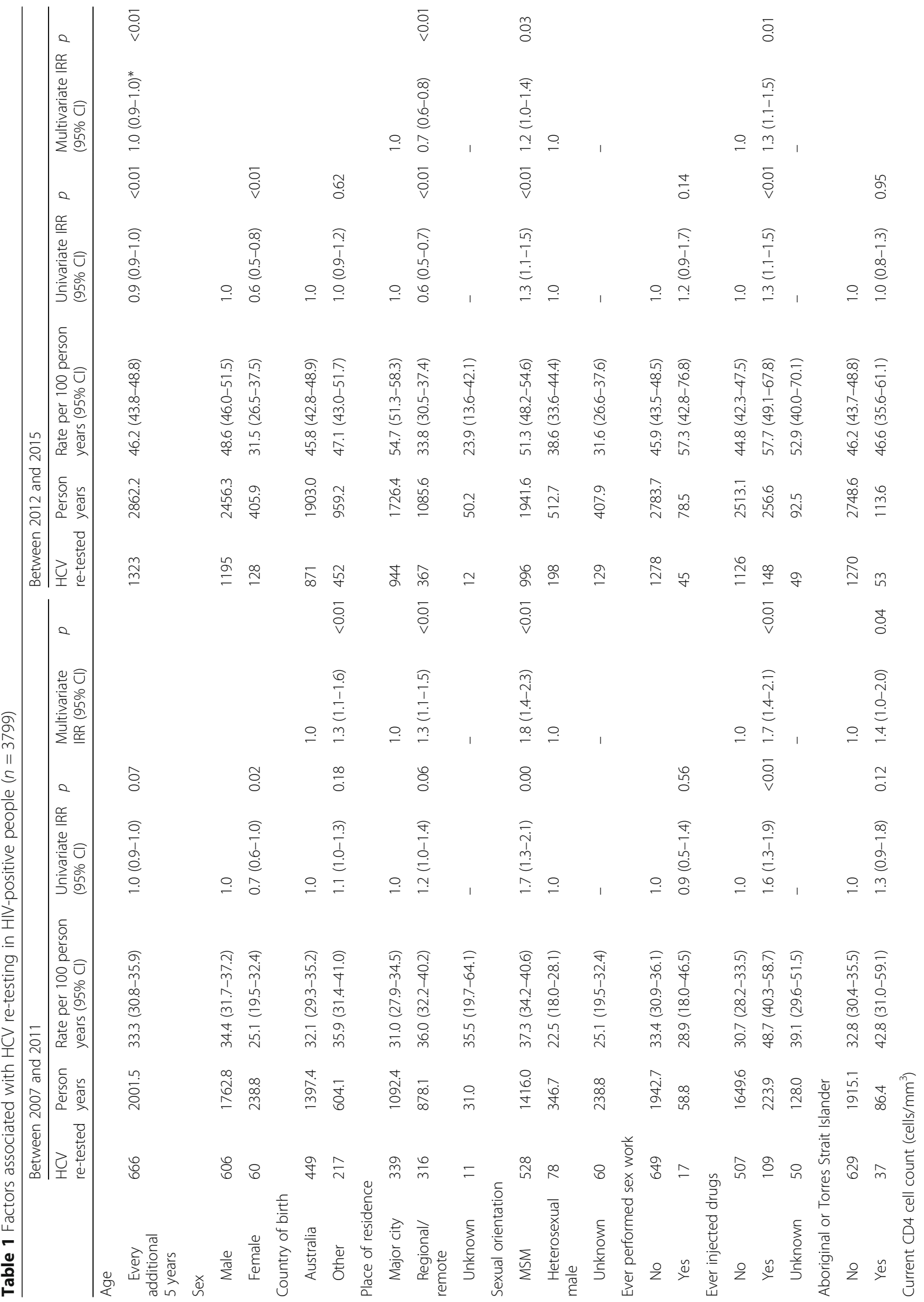


Boettiger et al. BMC Infectious Diseases (2017) 17:740

Page 7 of 12

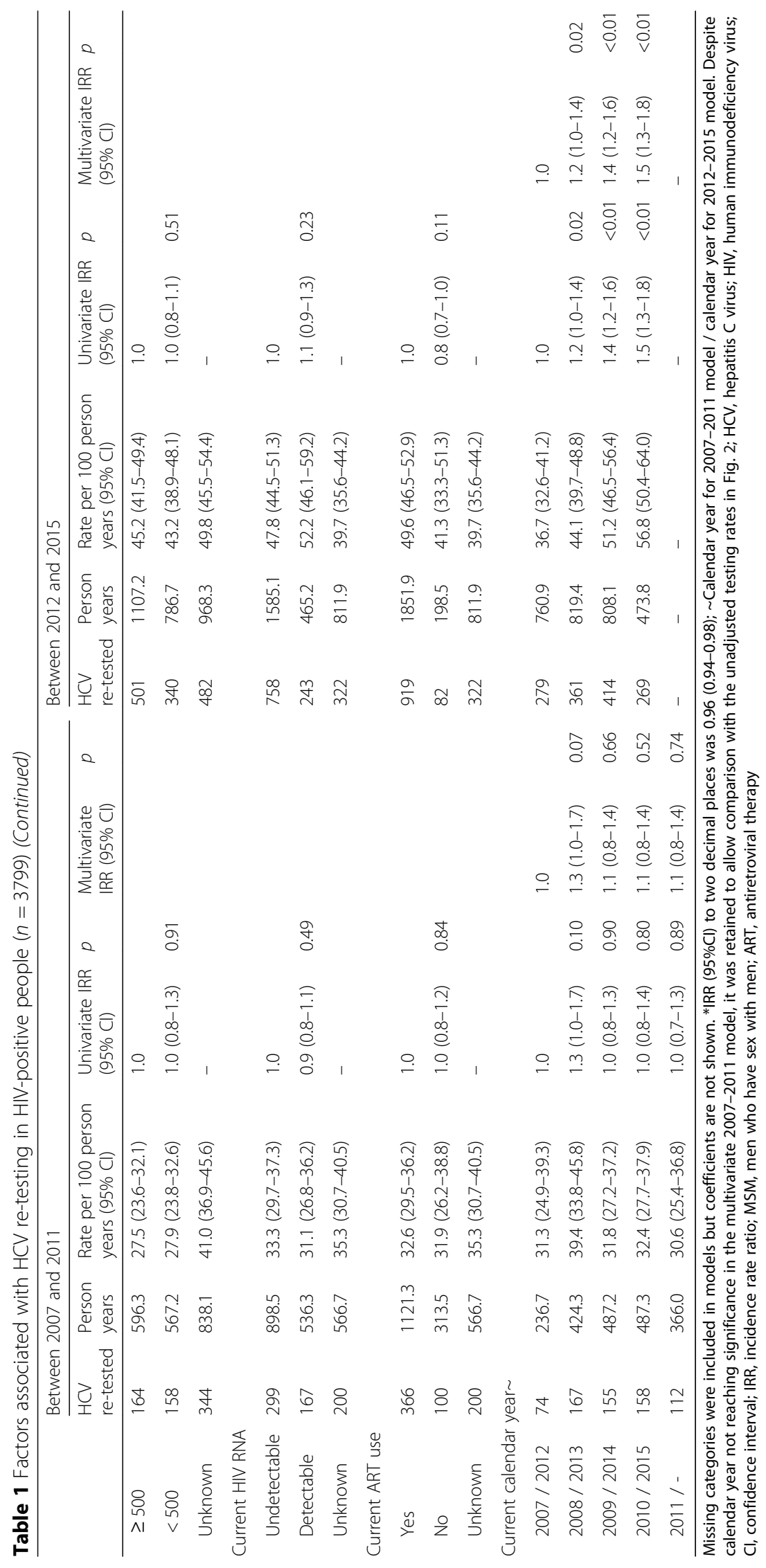


Boettiger et al. BMC Infectious Diseases (2017) 17:740

Page 8 of 12

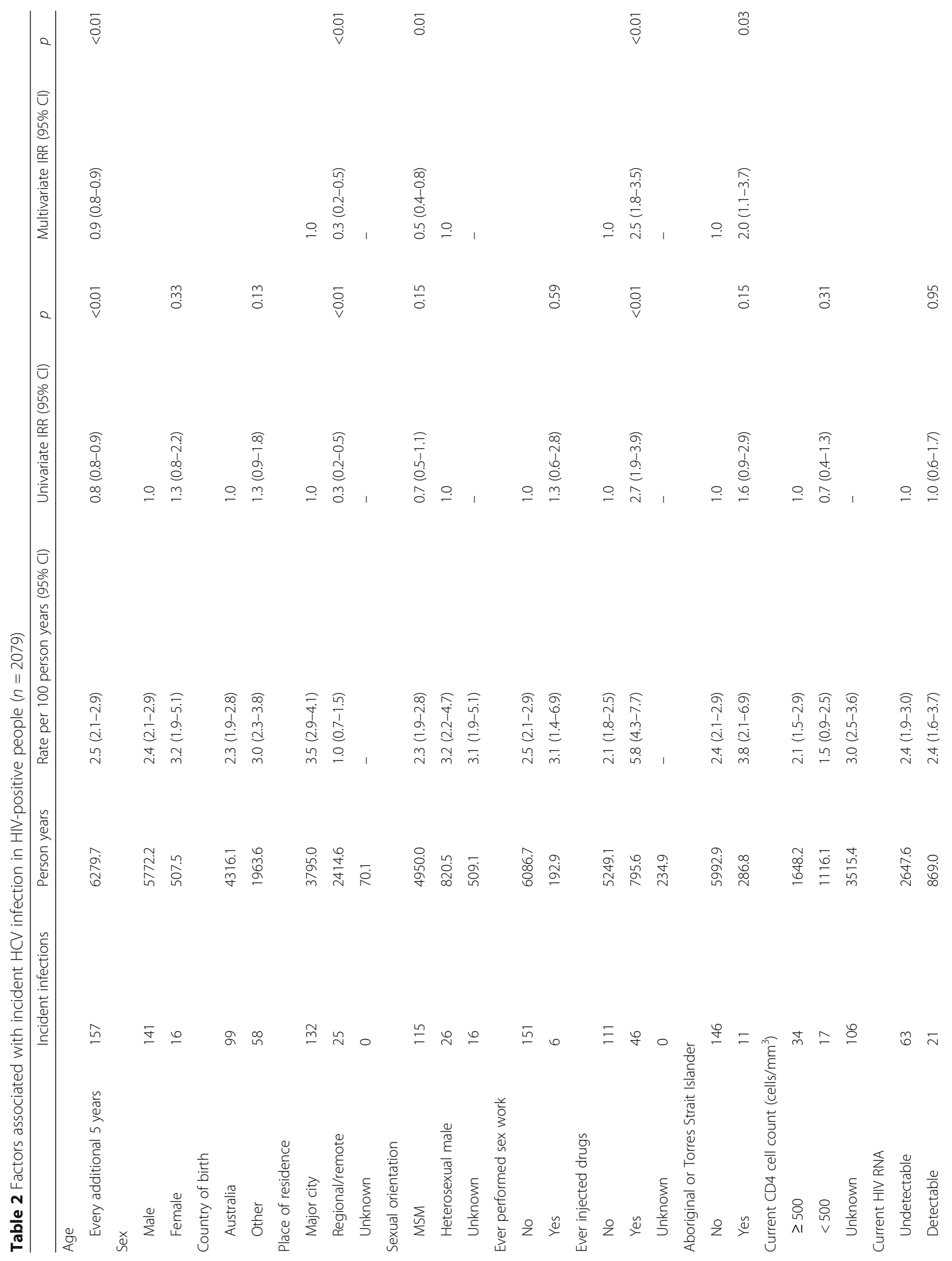







drug users between 2007 and 2015, however, there was a marked increase in testing rates during the latter years of the study period among those residing in a major city. The rate of incident $\mathrm{HCV}$ infection increased between 2007 and 2013 but remained stable between 2014 and 2015. Incident HCV was associated with a history of injecting drug use, particularly among heterosexual males.

HIV-positive MSM have an increased risk of $\mathrm{HCV}$ infection compared with HIV-negative MSM, possibly due to higher rates of high-risk sexual behavior [14]. Current Australian guidelines recommend routine HCV testing among HIV-positive MSM [15]. This explains why $\mathrm{HCV}$ testing and re-testing rates were consistently higher over time among our HIV-positive group. The increasing rates of HCV testing and re-testing observed are probably linked to a growing awareness of sexual HCV transmission [16] and, more recently, increasing awareness of DAA availability [17]. The number of state and national campaigns aiming to increase $\mathrm{HCV}$ and DAA awareness has increased substantially over the past decade $[18,19]$. Moreover, national advisory groups such as the Australasian Society for HIV, Viral Hepatitis and Sexual Health Medicine have actively sought to keep prescribers informed about DAA availability [20]. Given that non-sterile injecting practices and sexual behaviors that increase the potential for exposure to blood increase the possibility of transmitting $\mathrm{HCV}$, it was unsurprising to find that injecting drug users and MSM were subject to high rates of $\mathrm{HCV}$ re-testing. Of note, however, the increase in $\mathrm{HCV}$ re-testing rates between the periods 2007-2011 and 2012-2015 among patients living in a major city was contrasted by a small decline in testing among those living in regional/remote locations. This suggests that knowledge of DAA availability may be lacking outside of Australia's main urban centers.

Other recent studies from Australia and abroad have described an overall HCV incidence rate in HIV-positive people similar to that reported here (2.5 events per 100 person years) [12, 13, 21-27]. In contrast to our findings, however, which show HCV incidence increased between 2007 and 2013 then plateaued, data from the Swiss HIV Cohort suggests HCV incidence has increased consistently since 2000 among MSM [13] and results from the UK Collaborative HIV Cohort indicate that $\mathrm{HCV}$ incidence remained stable between 2004 and 2011 among MSM [12]. There are two important methodological differences between these two studies and ours. Firstly, unlike our study, patients enrolled in the Swiss and UK cohorts underwent routine HCV testing. The low rates of testing/re-testing during the earlier years of our study period could have caused us to underestimate $\mathrm{HCV}$ incidence. However, given $\mathrm{HCV}$ diagnoses were uncommon and that all patients in our incidence analysis were tested at least twice for $\mathrm{HCV}$, it is unlikely that testing rates substantially influenced our results. The other main difference is that the Swiss and UK studies defined incident infection as HCV seroconversion. Unfortunately, a preliminary assessment of our data indicated that, consistent with findings from the US [11], HCV testing was often not performed as recommended. This meant that the group of patients we identified as having experienced an incident HCV infection included an unknown number of re-infected patients. Nevertheless, our main and sensitivity analyses showed similar trends in both HCV testing and incident infection rates.

Interestingly, another Australian study recently found no change in HCV incidence between 2008 and 2016 among MSM [21]. Of note, this single-center study specifically asked HCV cases about injecting drug use and excluded those reporting a positive history of injecting regardless of what was noted in their records. Our analysis relied upon routinely collected surveillance data in which injecting drug use was probably underreported. This is supported by the results of our incidence model. While those with a history of injecting drug use had a high risk of incident HCV infection, it is likely that the other significantly associated factors (younger age, living in a major city, being a heterosexual male, and being Aboriginal and Torres Strait Islander) were largely the result of residual confounding. Each of these additional factors are known to be associated with injecting drug use $[28,29]$. This implies that the increase in HCV incidence among Australia's HIV-positive population may have been driven by injecting drug use and that sexual transmission has not changed substantially. This could be related to increased rates of unsafe injecting among sub-populations of the HIV-positive community. For example, between 2011 and 2014, the proportion of HIV infections attributable to IDU increased dramatically among Aboriginal and Torres Strait Islanders [5]. Importantly, heterosexual males that inject drugs appear to be at especially high risk of contracting $\mathrm{HCV}$. This finding warrants further investigation.

There are several limitations to bear in mind when evaluating the results of this work. Firstly, we were unable to link records between the sexual health services involved. Therefore, patients could have re-entered the analysis at multiple services. Additionally, we had limited information on sexual risk behavior and there is a high likelihood that injecting drug use was under-reported. Our analysis populations represent only a sample of the approximately 21,000 HIV patients who are currently diagnosed and in care in Australia [5], which means our rate estimates reflect only a subset of the total number of tests and diagnoses carried out nationwide. As mentioned earlier, an analysis of HCV testing and incidence across both sexual health services and general 
practice clinics involved in ACCESS is currently being planned. We were not able to conduct an appropriate analysis of factors associated with $\mathrm{HCV}$ testing because of the bias introduced by high-risk individuals knowing their $\mathrm{HCV}$ status prior to enrolment and therefore not undergoing testing. Further details on HCV testing rates and factors associated with HCV testing should form the topic of future investigation. Finally, while our testing and re-testing rate sensitivity analyses supported our main findings, our incidence rate sensitivity analyses were impeded by a very low number of outcomes.

\section{Conclusions}

The increasing rates of $\mathrm{HCV}$ testing and re-testing reported here are encouraging. High rates of $\mathrm{HCV}$ testing and re-testing among high-risk groups in Australia will assist strategies to achieve HCV elimination through rapid treatment scale up. Continued monitoring of $\mathrm{HCV}$ incidence in the HIV-positive population, particularly those with a history of injecting drug use, is essential for guiding both $\mathrm{HCV}$ prevention and treatment strategies.

\section{Additional file}

Additional file 1: Table S1. Characteristics of the HIV-positive and HIVnegative HCV testing rate populations at the time of their first clinic visit. Values are presented as $\mathrm{n}$ (\% of patients) unless otherwise indicated. *2073 individuals HIV seroconverted during follow up and contributed person years to both HIV-positive and HIV-negative study populations; HIV, human immunodeficiency virus; HCV, hepatitis $C$ virus; IQR, interquartile range; MSM, men who have sex with men. Table $\mathbf{S 2}$. Characteristics of the HIV-positive and HIV-negative HCV re-testing rate populations at the time of their first documented HCV test. Values are presented as n (\% of patients) unless otherwise indicated. *316 individuals HIV seroconverted during follow up and contributed person years to both HIV-positive and HIV-negative study populations; HIV, human immunodeficiency virus; HCV, hepatitis C virus; IOR, interquartile range: MSM, men who have sex with men. Table S3. Characteristics of the HCV incidence population at the time of their first negative HCV test. Values are presented as $\mathrm{n}$ (\% of patients) unless otherwise indicated. $\mathrm{HCV}$, hepatitis $\mathrm{C}$ virus; IQR, interquartile range; MSM, men who have sex with men; HIV, human immunodeficiency virus; ART, antiretroviral therapy. (DOCX $34 \mathrm{~kb})$

\section{Abbreviations}

ACCESS: Australian Collaboration for Coordinated Enhanced Sentinel Surveillance; Cl: Confidence interval; DAA: Direct acting antiviral; HCV: Hepatitis C Virus; HIV: Human Immunodeficiency Virus; IQR: Interquartile range; IRR: Incidence rate ratio; MSM: Men who have sex with men

\section{Acknowledgements}

The authors would like to acknowledge the participants that contribute data to ACCESS.

\section{Consent to publication}

Not applicable

\section{Funding}

This work was supported by a grant awarded to DCB by Gilead Sciences. Gilead Sciences played no role in the design of the study, data collection, data analysis, interpretation of data, or in the writing of the manuscript.

\section{Availability of data and materials}

The dataset analysed during the current study is not publicly available so as to maintain patient privacy. Data may be made available upon the receipt of reasonable requests. Please contact Denton Callander

(dcallander@kirby.unsw.edu.au) for further information.

\section{Authors' contributions}

DCB conceived and carried out the analysis, and drafted the final manuscript MGL, GJD, RG, DC, BD, CCOC, CKF, MH and GM participated in the design of the analysis and drafting of the final manuscript. All authors have read and approved the final manuscript.

\section{Ethics approval and consent to participate}

Approval for the sexual health services network of ACCESS was granted by the Human Research Ethics Committees of St Vincent's Hospital, Sydney (reference number 08/051) with further approval granted by the separate Human Research Ethics Committees responsible for each participating service. De-identified demographic, behavioral, pathology and diagnoses data were extracted from patient management systems using customized extraction scripts. Therefore, individual patient consent was waived.

\section{Competing interests}

DCB has received grant funding from Gilead Sciences. MGL has received unrestricted grants from Boehringer Ingelhiem, Gilead Sciences, Merck Sharp \& Dohme, Bristol-Myers Squibb, Janssen-Cilag, ViiV HealthCare, and consultancy fees from Gilead Sciences, and DSMB sitting fees from Sirtex Pty Ltd. GJD is an advisory board member and receives honorarium from Gilead Sciences, Merck Sharpe \& Dohme, Abbvie, and Bristol-Myers Squibb, has received research grant funding from Gilead Science, Merck Sharp and Dohme, Abbvie, and Bristol-Myers Squibb, and travel sponsorship from Gilead Science, Merck Sharp and Dohme, Abbvie, and Bristol-Myers Squibb. MH has received funding for investigator initiated research from Gilead Sciences, Bristol-Myers Squibb and Abbvie. GM has received research funding and consultancy fees for Gilead Sciences and Abbvie. All other authors report no competing interests.

\section{Publisher's Note}

Springer Nature remains neutral with regard to jurisdictional claims in published maps and institutional affiliations.

\section{Author details}

${ }^{1}$ The Kirby Institute, UNSW Sydney, Sydney, NSW, Australia. ${ }^{2}$ Sydney Sexual Health Centre, Sydney, NSW, Australia. ${ }^{3}$ The Kirby Institute, UNSW Sydney and Sexual Health Service, Sydney Local Health District, Sydney, NSW Australia. ${ }^{4}$ Central Clinical School, Sydney University, Sydney, NSW, Australia. ${ }^{5}$ Melbourne Sexual Health Centre, Alfred Health and Monash University, Melbourne, Victoria, Australia. ${ }^{6}$ Viral Hepatitis Elimination Program, Burnet Institute, Melbourne, Victoria, Australia.

Received: 17 September 2017 Accepted: 22 November 2017 Published online: 01 December 2017

\section{References}

1. Greub $G$, Ledergerber B, Battegay M, Grob P, Perrin L, Furrer $H$, Burgisser $P$, Erb P, Boggian K, Piffaretti JC, et al. Clinical progression, survival, and immune recovery during antiretroviral therapy in patients with HIV-1 and hepatitis C virus coinfection: the Swiss HIV Cohort Study. Lancet. 2000; 356(9244):1800-5

2. Sulkowski MS, Mast EE, Seeff LB, Thomas DL. Hepatitis C virus infection as an opportunistic disease in persons infected with human immunodeficiency virus. Clin Infect Dis. 2000;30(Suppl 1):S77-84.

3. Thein $H_{1}$, Yi Q, Dore GJ, Krahn MD. Natural history of hepatitis C virus infection in HIV-infected individuals and the impact of HIV in the era of highly active antiretroviral therapy: a meta-analysis. AIDS. 2008;22(15):1979-91.

4. The Australian HIV Observational Database Annual report 2016.

5. The Kirby Institute. HIV, viral hepatitis and sexually transmissible infections in Australia. Annual Surveillance Report 2017. The Kirby Institute, UNSW Australia, Sydney NSW 2052.

6. Arends JE, Lieveld FI, Boeijen LL, de Kanter CT, van Erpecum KJ, Salmon D, Hoepelman Al, Asselah T, Ustianowski A. Natural history and treatment of 
HCV/HIV coinfection: is it time to change paradigms? J Hepatol. 2015;63(5): 1254-62.

7. Australian Government: Turnbull govt invests over $\$ 1 B$ to cure Hep C - media release. (2015)

8. Gastroenterological Society of Australia: Australian recommendations for the management of hepatitis C virus infection: a consensus statement (2017). Available from: https://www.asid.net.au/documents/item/1208 [accessed: 08 Mar 2017].

9. Aitken S. ASHM Report Back - Elimination of Hepatitis C and HIV coinfection in Australia. Available from: http://ashm.info/hiv-co-infections/elimination-ofhepatitis-c-and-hiv-coinfection-in-australia [accessed: $10 \mathrm{Mar} 2017$ ].

10. Guy RJ, Kong F, Goller J, Franklin N, Bergeri I, Dimech W, Reilly N, Sullivan E, Ward J, Kaldor JM, et al. A new national Chlamydia sentinel surveillance system in Australia: evaluation of the first stage of implementation. Commun Dis Intell Q Rep. 2010;34(3):319-28.

11. Centers for Disease Control and Prevention. Hepatitis C. Available from: https:/ www.cdc.gov/vitalsigns/hepatitisc/index.html [accessed: 08 Mar 2017].

12. Martin NK, Thornton A, Hickman M, Sabin C, Nelson M, Cooke GS, Martin TC, Delpech V, Ruf M, Price $\mathrm{H}$, et al. Can hepatitis C virus (HCV) direct-acting antiviral treatment as prevention reverse the HCV epidemic among men who have sex with men in the United Kingdom? Epidemiological and modeling insights. Clin Infect Dis. 2016;62(9):1072-80.

13. Salazar-Vizcaya L, Kouyos RD, Zahnd C, Wandeler G, Battegay M, Darling KE, Bernasconi E, Calmy A, Vernazza P, Furrer $\mathrm{H}$, et al. Hepatitis $\mathrm{C}$ virus transmission among human immunodeficiency virus-infected men who have sex with men: modeling the effect of behavioral and treatment interventions. Hepatology. 2016;64(6):1856-69.

14. van de Laar TJ, Matthews GV, Prins M, Danta M. Acute hepatitis C in HIVinfected men who have sex with men: an emerging sexually transmitted infection. AIDS. 2010;24(12):1799-812.

15. National HCV Testing Policy Expert Reference Committee. National Hepatitis C Testing Policy v1.2. Available from: http://testingportal.ashm.org.au/ images/HCV/HCV_TESTING_POLICY_Mar_2017.pdf [accessed: 03 Nov 2017].

16. Jin FY, Matthews GV, Grulich AE. Sexual transmission of hepatitis $C$ virus among gay and bisexual men: a systematic review. Sex Health. 2017; 14(1):28-41.

17. Higgs P, Hsieh K, Hellard M. "You're better off waiting": knowledge and awareness of hepatitis $C$ direct-acting antivirals in a cohort of people who inject drugs (P1269). J Hepatol. 2015;62:S834.

18. Hepatitis Australia. Love your liver. Available from: http://loveyourliver.com au/ [accessed: 06 Nov 2017].

19. New South Wales Health. Enter the party. Available from: http://hepc.health. nsw.gov.au/ [accessed: 06 Nov 2017].

20. ASHM. Australasian Society for HIV, Viral Hepatitis and Sexual Health Medicine. Available from: http://www.ashm.org.au/ [accessed: 06 Nov 2017].

21. Medland NA, Chow EP, Bradshaw CS, Read TH, Sasadeusz JJ, Fairley CK. Predictors and incidence of sexually transmitted hepatitis $C$ virus infection in HIV positive men who have sex with men. BMC Infect Dis. 2017;17(1):185.

22. Hagan $\mathrm{H}$, Jordan $A E$, Neurer J, Cleland CM. Incidence of sexually transmitted hepatitis $\mathrm{C}$ virus infection in HIV-positive men who have sex with men. AIDS. 2015;29(17):2335-45.

23. Wandeler G, Gsponer T, Bregenzer A, Gunthard HF, Clerc O, Calmy A, Stockle $\mathrm{M}$, Bernasconi E, Furrer $\mathrm{H}$, Rauch $\mathrm{A}$, et al. Hepatitis $\mathrm{C}$ virus infections in the Swiss HIV cohort study: a rapidly evolving epidemic. Clin Infect Dis. 2012;55(10):1408-16

24. Gamage DG, Read TR, Bradshaw CS, Hocking JS, Howley K, Chen MY, Fairley CK. Incidence of hepatitis-C among HIV infected men who have sex with men (MSM) attending a sexual health service: a cohort study. BMC Infect Dis. 2011;11:39.

25. Vanhommerig JW, Stolte IG, Lambers FA, Geskus RB, van de Laar TJ, Bruisten SM, Schinkel J, Prins M. Stabilizing incidence of hepatitis $C$ virus infection among men who have sex with men in Amsterdam. J Acquir Immune Defic Syndr. 2014;66(5):e111-5.

26. Witt MD, Seaberg EC, Darilay A, Young S, Badri S, Rinaldo CR, Jacobson LP, Detels $R$, Thio $C L$. Incident hepatitis $C$ virus infection in men who have sex with men: a prospective cohort analysis, 1984-2011. Clin Infect Dis. 2013; 57(1):77-84.

27. van Santen DK, van der Helm JJ, Del Amo J, Meyer L, D'Arminio Monforte A, Price M, Beguelin CA, Zangerle R, Sannes M, Porter K, et al. Lack of decline in hepatitis $C$ virus incidence among HIV-positive men who have sex with men during 1990-2014. J Hepatol. 2017;67(2):255-62.
28. Memedovic S, Iversen J, Geddes L, Maher L. Australian Needle Syringe Program Survey National Data Report 2011-2015: Prevalence of HIV, HCV and injecting and sexual behaviour among NSP attendees. Available from: https://kirby.unsw.edu.au/report-type/australian-nsp-survey-national-datareport. [accessed: 09 Mar 2017].

29. Sundquist K, Frank G. Urbanization and hospital admission rates for alcohol and drug abuse: a follow-up study of 4.5 million women and men in Sweden. Addiction. 2004;99(10):1298-305.

\section{Submit your next manuscript to BioMed Central and we will help you at every step:}

- We accept pre-submission inquiries

- Our selector tool helps you to find the most relevant journal

- We provide round the clock customer support

- Convenient online submission

- Thorough peer review

- Inclusion in PubMed and all major indexing services

- Maximum visibility for your research

Submit your manuscript at www.biomedcentral.com/submit
Biomed Central 